Supporting Information

\title{
Unique Gelation of Polyethylene Glycol-Modified Lignin in Hot Ethanol and Its Application to Synthesis of Epoxy Resin with Large Lignin Content
}

Osamu Tanaike*, Kenta Ono, Ryo Ishii, Takeo Ebina

Research Institute for Chemical Process Technology, National Institute of Advanced Industrial Science and Technology (AIST), 4-2-1 Nigatake Miyagino-ku, Sendai, Miyagi 983-8551, Japan

Shiho Takahashi, Thi Thi Nge, Tatsuhiko Yamada

Center for Advanced Materials, Forestry and Forest Products Research Institute (FFPRI),1 Matsunosato, Tsukuba, Ibaraki 305-8687, Japan

*Corresponding author: Osamu Tanaike

E-mail: o-tanaike@aist.go.jp 


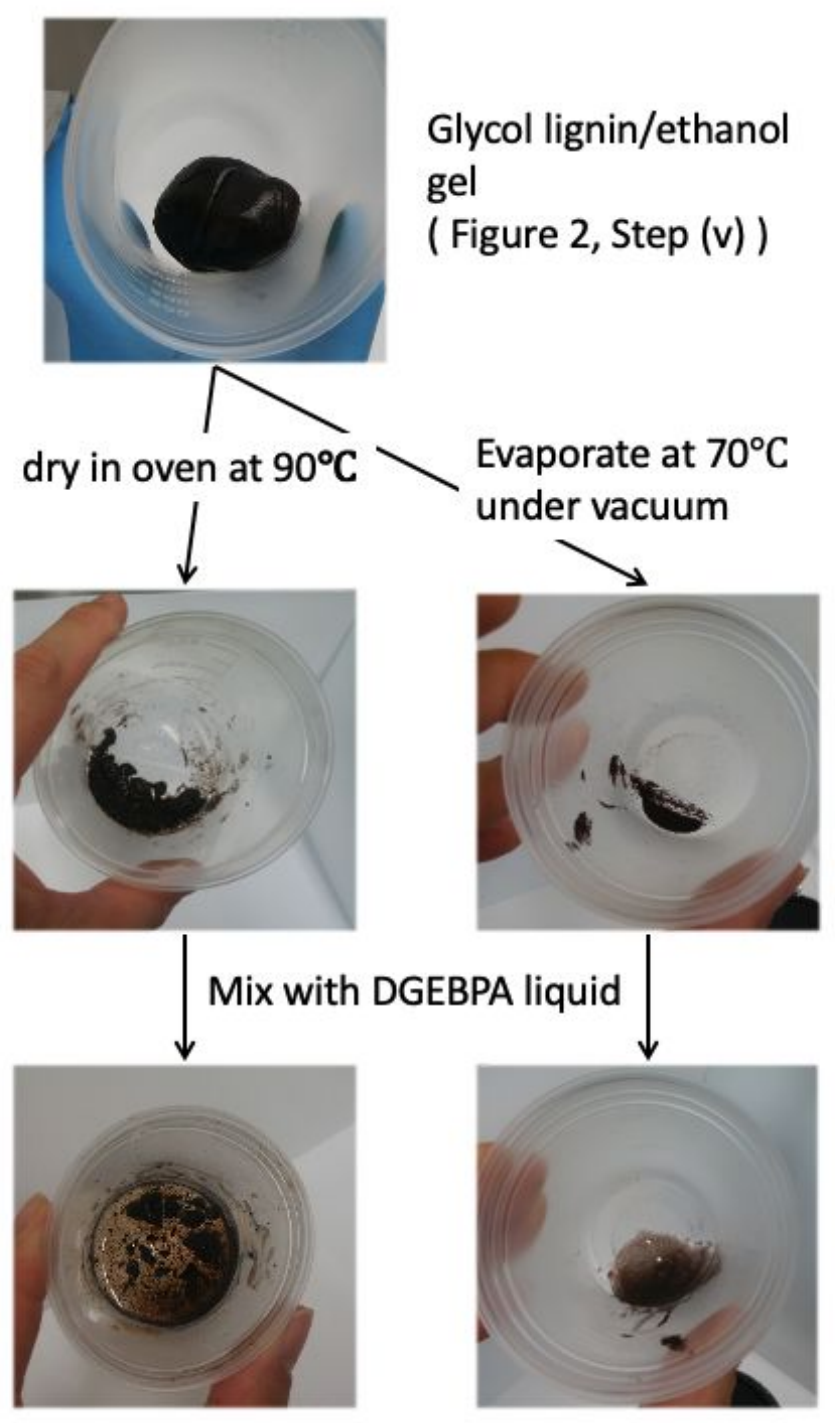

Figure S1. Comparison of the appearance of aggregated glycol lignin soft mass in ethanol when mixed with the epoxy main agent after ethanol removal by two different methods. In both cases, the glycol lignin and DGEBPA were separated without mix. 




(a)

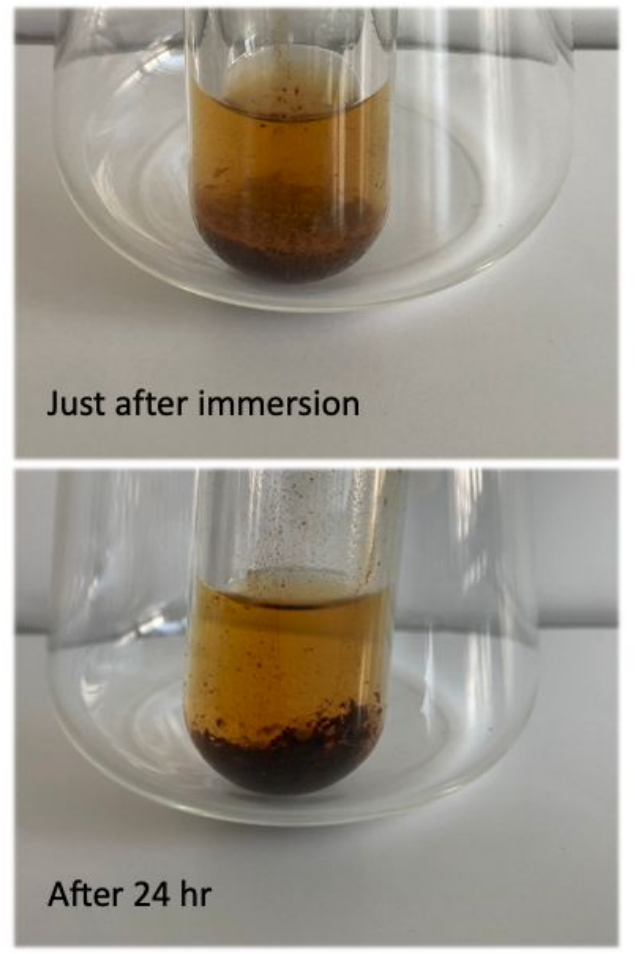

(b)

Figure S2. (a) Comparison of the dispersion and aggregation behavior of glycolic lignin and

sodium lignin sulfonate in ethanol at room temperature, and (b) magnified views of glycol lignin

before and after aggregation at room temperature. 


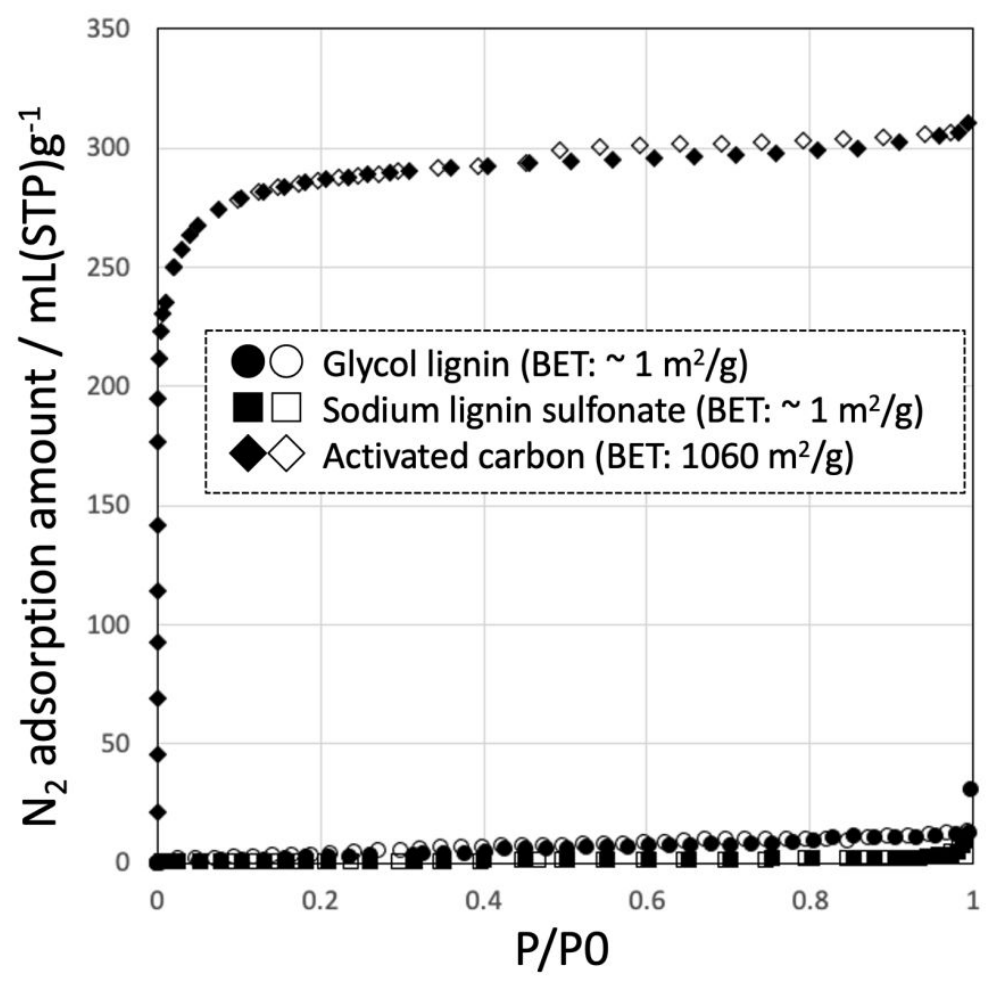

Figure S3. Nitrogen adsorption/desorption isotherms at $-196^{\circ} \mathrm{C}$ of glycol lignin, sodium lignin sulfonate, and activated carbon to confirm their porosities. The closed and open marks indicate adsorption and desorption, respectively. 
